individual basis, requires physician referral and cannot exceed 2 hours of training per year for a beneficiary, plus 1 hour of nutritional services. We propose to study a novel and more cost-effective approach to improving self-efficacy and outcomes for patients with established type 2 diabetes who are not achieving optimal glycemic control. This randomized trial will formally evaluate the impact of a conversational group-based experience called IDEA (Interactive Dialogue Educational Activity). Methods: This multi-site, randomized, prospective trial will randomize 621 patients with A1c $>7 \%$ to one of 3 study groups: (1) IDEA, 244 patients receive group sessions totaling 8 hours, with an average of 10 patients per group, using conversation maps as a conduit to facilitate dialogue between providers and patients; (2) Individual Education (IE), 244 patients receive 2 hours with a nurse educator and 1 hour with a dietitian; and (3) Usual care (UC), 133 patients receive no research intervention. The primary analyses will involve mixed model regression to assess whether IDEA improves outcomes compared to IE and UC. A1c, blood pressure, and lipids will be evaluated 6 and 12-months post-randomization, and behavioral, emotional, and satisfaction outcomes will be evaluated through survey at baseline, 3, 6, 9 and 12 months. Depending on 12-month results, a longitudinal four year post-intervention analysis is planned to assess sustainability of treatment effects and cost-effectiveness. Results: Results of this Merck funded trial, expected by June of 2010, could shed landmark evidence on whether education emphasizing interactive group activities can improve diabetes outcomes for patients who are not achieving optimal glycemic control. In addition, the study will assess whether this type of group experience is more effective than more traditional and costly individual education. Conclusions: Better ways are needed to improve diabetes patient self-management behaviors and outcomes. This rigorously designed study will contribute to knowledge necessary to determine the best content and style of delivery for diabetes education.

\section{Abstract PS1-17 \\ Husbands' Support and Women's Psychosocial Adjustment to an Ostomy Resulting from Colorectal Cancer}

Andrea Altschuler, PhD, Kaiser Permanente Division of Research; Michelle Ramirez, PhD, University of the Sciences in Philadelphia; Marcia Grant, RN, DNSc, City of Hope Cancer Center; Carmit McMullen, PhD, The Center for Health Research, Northwest; Mark Hornbrook, PhD, The Center for Health Research, Northwest; Lisa Herrinton, PhD, Kaiser Permanente Division of Research; Robert Krouse, MD, University of Arizona

Background/Aims: Some colorectal cancer (CRC) patients require a permanent ostomy (a surgically created opening on the exterior of the abdomen that allows feces to be eliminated from the body). An ostomy can create psychosocial distress for women and affect their body image, sense of self, and sexual functioning. Little is known, however, about how a husband's (or long-term male partner's) support influences their wife's psychosocial recovery. Methods: In this multi-site, NCI-funded study of long-term CRC survivors, participants completed the modified City of Hope-Quality of Life questionnaire, which yields scores of health-related quality of life (HR-QOL). We then conducted in-depth interviews with 30 female participants covering the topics of body image; sexuality in terms of personal history; meanings of sexuality practices and desires; and information needs regarding sexuality. Interviews were tape recorded, transcribed, and analyzed using standard qualitative methods. We compared women's global HR-QOL survey scores to interview discussions about husbands' support and psychosocial adjustment to an ostomy. Results: Of the 30 interviewees, 22 were married/partnered at the time of surgery or shortly thereafter. Of the 22 married/partnered women, 17 described positive support from their husbands as key to psychosocial adjustment, 3 described how a lack or withdrawal of support impeded recovery, and 2 described their husbands' support as neither positive nor negative. Among the 20 women who expressed either a positive or negative influence of their husbands' support, correlations with HR-QOL were noted. In 17 cases, women's high or low HR-QOL scores matched the positive or negative qualitative findings. The remaining 3 cases had positive qualitative data and low HR-QOL scores, but each of these women had serious current comorbidities. Conclusions: Husbands'/partners' support or lack thereof can have considerable short-term and long-term influences on the psychosocial recovery of female CRC patients with ostomies. Future research could validate these findings in a larger sample.
Abstract PS1-18

The Effect of No Tube on Gastric Emptying in Patients Undergoing Pancreatic Surgery

Christie C. Cummings, RN, MA, Scott \& White; Staci Hix-Hernandez, MD, Scott \& White; Mohsen M. Shabahang, MD, Scott \& White; M. Hasan Rajab, $\mathrm{PhD}$, Scott \& White

Background: Delayed gastric emptying occurs in about 30\% of patients undergoing pancreaticoduodenectomy. Tube feedings have been thought to exacerbate this problem. The main objective is to determine the effects of not performing enteral feedings via jejunostomy tube after pancreatic resection. Methods: We conducted an IRB approved, clinical trial in patients undergoing pancreaticoduodenectomy or total pancreatectomy. Patients were randomized to either a jejunostomy feeding tube placed at the time of surgery with tube feedings started 48 hours later followed by an oral diet on postoperative day 5 (Group A), or no feeding tube and started on an oral diet on postoperative day 5 (Group B). Drainage from the nasogastric tube, need for parenteral nutrition, and days until discharge were compared. We used t-test or non-parametric Wilcoxon test for comparing the main variables using one-sided test with a 0.05 level of significance. Results: Twenty-seven patients were included in the study: 15 in Group A, and 12 in Group B. The groups were similar in baseline characteristics except average age; 69 years in Group A vs 58 years in Group B $(P=0.015)$. Comorbidities and indications for surgery were not significantly different between the two groups. In addition, 24 of 27 (89\%) patients had cancer. The majority of patients had the nasogastric tube for 4 days with no significant difference seen in the output volumes between the two groups. Despite the enteral nutrition, $54 \%$ of the patients in Group A required parenteral nutrition compared to $25 \%$ in Group B $(P=0.23)$. The average number of hospital days was 19.6 for Group A, and 9.1 for Group B $(P=0.06)$. Conclusions: Patients tend to do better on no enteral feedings via jejunostomy tube after pancreatic resection. This finding has been translated into practice at our institution and patients resume oral intake postoperatively without jejunostomy tube feedings.

\section{Abstract PS1-19 \\ Improvements in Colorectal Cancer Screening Among Medicare Enrollees, 2000-2005}

Chyke A. Doubeni, MD, MPH, University of Massachusetts Medical School/ Meyers Primary Care Institute; Terry S. Field, ScD, Meyers Primary Care Institute/University of Massachusetts Medical School

Background: Medicare expanded coverage of colorectal cancer (CRC) screening in 2001 to include reimbursement for screening colonoscopy. The objective of this report was to examine trends and factors associated with CRC screening among non-institutionalized Medicare beneficiaries aged 65-80 years from 2000-2005. Methods: We used data from the Medicare Current Beneficiary Surveys for 2000, 2003 and 2005 linked to Medicare claims data. The primary outcome for this study was the proportion who were up-to-date for CRC screening, defined as undergoing home fecal occult blood testing (FOBT) within one year and/or colonoscopy within five years of the interview date. We used the Chi-square test for trend to assess changes over the six-year period under study and examined predictors of screening using multivariable logistic regression models. Results: The characteristics of the participants were similar for all three study-years. For 2000, median age was 73 years; $57 \%$ were female; $81 \%$ were whites, $9 \%$ were blacks, $7 \%$ were Hispanics, and $4 \%$ other ethnicities; $37 \%$ had income $>\$ 25,000$; and $33 \%$ had $8^{\text {th }}$ grade or less education, $35 \%$ up to high school graduation and $31 \%$ were educated beyond high school. During the study period, there was a statistically significant increase in the proportion of beneficiaries undergoing CRC screening from $44 \%$ to $51 \%$. FOBT rates decreased from $18 \%$ to $14 \%$ while colonoscopies increased from $36 \%$ to $57 \%$. These changes were all statistically significant. For each of the three interview-years, enrollees were less likely to have been screened if they had not heard about CRC screening, did not know that Medicare provided reimbursement, a physician did not recommend testing, were lower income, less educated populations and experienced difficulty in receiving healthcare. Conclusions: CRC screening among Medicare beneficiaries increased significantly from 2000 to 2005, following the introduction of expanded coverage. This increase occurred in tandem with increasing use of colonoscopy among beneficiaries. However, efforts are still needed to increase the reach of CRC to disadvantaged populations. 